\title{
HIPNOSIS, MAGNETISMO ANIMAL Y MONSTRUOSIDAD EN LA LITERATURA INGLESA DE FINALES DEL SIGLO XIX
}

\author{
Juan Marcos Bonet Safont \\ Universidad de Valencia \\ Email: bonetsafont@gmail.com \\ ORCID iD: http://orcid.org/0000-0002-9614-0205
}

Recibido: 28 junio 2018; Aceptado: 12 abril 2019.

Cómo citar este artículo/Citation: Bonet Safont, Juan Marcos (2019), “Hipnosis, magnetismo animal y monstruosidad en la literatura inglesa de finales del siglo XIX", Asclepio, 71(2): p279. https://doi.org/10.3989/asclepio.2019.20

RESUMEN: En el presente trabajo exploraremos la imagen literaria del magnetismo animal y de la hipnosis a través del análisis de dos obras de ficción: las novelas El escarabajo (1897) de Richard Marsh y Drácula (1897) de Bram Stoker. Durante todo el siglo $\mathrm{XIX}$, aunque principalmente a finales de este, fueron muchos los autores que recurrieron al magnetismo animal y la hipnosis, de forma ambiental o argumental, en sus creaciones de ficción, tanto fue así que Arthur Quiller-Couch, un importante crítico literario del siglo XIX, llegó a hablar del surgimiento de un nuevo subgénero literario que acuñó como "hypnotic fiction". Partiendo de la idea de que en esta literatura de ficción mesmérica e hipnótica se pueden rastrear claramente unos estereotipos diferenciados de magnetizadores e hipnotizadores (unos más clásicos que otros) quienes hacen un uso diverso e incluso antagónico del magnetismo animal y de la hipnosis, mostraremos la relación de estos conocimientos con la figura del monstruo en las creaciones de Richard Marsh y Bram Stoker y como estos no pueden reducirse al estereotipo de magnetizador/hipnotizador villano, ya que los poderes mesméricos e hipnóticos funcionan aquí como agregados para conformar la monstruosidad del personaje.

PALABRAS CLAVE: magnetismo animal; hipnosis; literatura de ficción; monstruos; El escarabajo; Drácula.

\section{HYPNOSIS, ANIMAL MAGNETISM, AND MONSTROSITY IN LATE NINETEENTH CENTURY ENGLISH LITERATURE}

ABSTRACT: We will explore the literary image of animal magnetism and hypnosis through the analysis of two works of fiction: the novels Richard Marsh's The Beetle: A Mystery (1897) and Bram Stoker's Dracula (1897). During all the 19th century and mainly at its last, many authors used animal magnetism and hypnosis in their fictional creations in an environmental or plot way, so much that Arthur Quiller-Couch, an important literary critic of the nineteenth century, spoke about the emergence of a new literary subgenre that he called "hypnotic fiction". Starting from the idea that in this mesmeric and hypnotic fiction literature you can clearly trace differentiated stereotypes of magnetizers and hypnotists (some of them more classic than others) which perform a diverse and even antagonistic use of animal magnetism and hypnosis, we will show the relationship of this knowledge with the figure of the monster in the creations of Richard Marsh and Bram Stoker and how these cannot be reduced to the stereotype of a villain magnetizer/ hypnotist, as mesmeric and hypnotic powers work here as aggregates to conform the monstrosity of the character.

KEY WORDS: Animal Magnetism; Hypnosis; Fiction Literature; Monsters; The Beetle; Dracula. mons Reconocimiento 4.0 Internacional (CC BY 4.0). 


\section{INTRODUCCIÓN}

Hablando de literatura inglesa es difícil encontrar una novela del período victoriano tardío en la que no aparezcan la hipnosis, la posesión, la telepatía, el mesmerismo o los médiums (Luckhurst, 2002, p. 182). Tanto fue así que Arthur Quiller-Couch, un importante crítico literario del siglo XIX, llegó a hablar del surgimiento de un nuevo subgénero literario que acuñó como "hypnotic fiction" (Quiller-Coach, 1890, p. 316). Mucho más tarde, fuera del campo de la crítica literaria y dentro de los estudios académicos, Roger Luckhurst ha denominado a estas creaciones literarias como "trance-gothic" (Luckhurst, 2000, p. 148) y Julian Wolfreys como "mesmeric texts" (Wolfreys, 2007, p. 13). Si se consulta el repertorio bibliográfico de Donald Hartman titulado Hypnotic and Mesmeric Themes and Motifs in Selected English-Language Novels, Shorts Stories, Plays and Poems, 1820-1983 (1987), comprobaremos que fue a finales del siglo XIX cuando se alcanzó la mayor producción de este tipo de literatura en lengua inglesa. También en la Francia fin de siècle se dio un auge sin precedentes de ficciones mesméricas, superando en el número de obras al caso inglés (Darnton, 1968, p. 151). El relato pionero de esta tradición narrativa es El magnetizador (1813) de E.T.A. Hoffmann, el más popular es La verdad sobre el caso del señor Valdemar (1845) de Edgar Allan Poe y el que mayor éxito de ventas consiguió en el momento de su publicación fue Trilby (1894) de George Du Maurier, considerado como el primer best-seller de la era moderna (Purcell, 1977, p. 62). En general, todas estas ficciones mesméricas reflejan un hecho esencial: la perversión de los ideales de Mesmer atravesados y contaminados por las bajas pasiones humanas encarnadas en la figura del magnetizador y del hipnotizador (Montiel, 2003, p. 157).

Es imprescindible, siquiera brevemente, esclarecer los diferentes términos (magnetismo animal, mesmerismo e hipnosis) usados por los novelistas en sus textos. El médico vienés Franz Anton Mesmer (17341815) denominó "magnetismo animal" a la radiación en forma de fluido universal que conecta todo con todo; mediante este fluido universal los seres humanos estarían conectados no solo con el resto de seres humanos sino con las plantas, los árboles, los animales, e incluso con los diferentes cuerpos astrales (Gauld, 1992, p. 11). Los autores del romanticismo alemán se sintieron fascinados por el magnetismo animal y lo utilizaron como tema central de sus creaciones literarias, siendo los que acuñaron y popula- rizaron el término "mesmerismo" (López-Piñero; Morales, 1970, p. 123). Por otra parte, el término "hipnosis" surge de las ideas del cirujano escocés James Braid (1795-1860), que llamó "hipnotismo" a la forma extrema del "sueño nervioso" que presentaba amnesia tras el despertar (López-Piñero, 2002, p. 49). Braid rechazó la teoría del fluido universal, y empleó una metodología totalmente diferente a la de Mesmer (Gauld, 1992, p. 281), por lo que puede decirse que el magnetismo animal y la hipnosis descansan sobre unas bases epistémicas y antropológicas totalmente diferentes, con visiones distintas y hasta contrapuestas de la medicina, la salud y la enfermedad. Sintetizando, entre el magnetismo animal y el mesmerismo hay diferencias de matiz, pero ambas concepciones permanecen cercanas entre sí, en cambio la hipnosis está muy lejos de ambas.

En general, aunque con interesantes excepciones como veremos más adelante, los escritores de ficción van a usar los tres términos (magnetismo animal, mesmerismo e hipnosis) para hablar de lo mismo: los poderes magnéticos dejan de ser un método curativo y se convierten en un método para subyugar al otro en un afán de acumular poder. En realidad, el propio método terapéutico de Mesmer, el famoso baquet que provocaba las llamadas crisis magnéticas, aparece escasamente reflejado en la literatura de ficción ${ }^{1}$. En cambio, sí que aparece, de forma notable, toda la parafernalia usada por Mesmer para ayudar a su cubeta a provocar las crisis; de este modo los ropajes exóticos con los que se disfrazaba, los ambientes a media luz y la música sugerente han pasado a formar parte de los topos literarios en las ficciones mesméricas e hipnóticas. Sin embargo, los métodos que aparecen en estas ficciones para conseguir el trance magnético, con escasas excepciones, son una mezcla de las técnicas empleadas por los magnetizadores (como los pases de manos) y por los hipnotizadores (como la fijación de la mirada en un punto o las inducciones verbales). Ni siquiera son los métodos del marqués de Puységur (1751-1825), descubridor del llamado "sonambulismo provocado" (López-Piñero, 2002, p. 34), aspecto despreciado por Mesmer y más tarde tenido en cuenta por los teóricos de la hipnosis, pues en las ficciones mesméricas el magnetizado o hipnotizado no entra en crisis, sino que duerme.

Casi todos los autores que, fascinados por el magnetismo animal y la hipnosis, lo han utilizado en sus relatos de ficción han explorado las posibilidades paranormales que rodean al tema y no sus posibilidades 
curativas. A partir de ahí, la literatura de ficción nos presenta un variado, aunque limitado, empleo del magnetismo animal y de la hipnosis que va conformando una pequeña galería de magnetizadores e hipnotizadores que, aunque reconocibles como tales, representan estereotipos diferentes. El argumento habitual es presentar el magnetismo animal y la hipnosis como poderes misteriosos mediante los cuales un villano consigue sus egoístas propósitos a través de la subyugación de la voluntad de la víctima; así ocurre, por ejemplo, en la novela Trilby (1894) de George Du Maurier (aunque en un primer momento el uso del magnetismo animal en esta novela es terapéutico) y en la novela inacabada de Charles Dickens El misterio de Edwin Drood (1870). También es bastante habitual encontrar al personaje del magnetizador/hipnotizador mezclado con elementos ocultos y/o esotéricos; en particular, con diversos topos literarios (personajes, escenarios, teorías y rituales) del ambiente espiritista, como ocurre en las novelas de BulwerLytton Zanoni (1842) y Una historia extraña (1862). Otro estereotipo habitual de magnetizador/hipnotizador en la literatura de ficción es el charlatán, aquel que comete fraude y engaña a sus víctimas, así ocurre en dos populares novelas de la época: Herr Paulus: His Rise, His Greatness, and His Fall (1888) de Walter Besant y The Charlatan (1895) de Robert Buchanan. Uno de los más interesantes estereotipos de magnetizador/hipnotizador es el "científico loco", aquel que lleva sus experimentos en torno al magnetismo animal y de la hipnosis más allá de toda ética y moral, dos ejemplos de este uso los observamos en La verdad sobre el caso del señor Valdemar (1845) de Edgar Allan Poe y El gran experimento de Keinplatz (1885) de Conan Doyle. $Y$, por último, en esta lista de magnetizadores e hipnotizadores podríamos situar al detective. Estos últimos usan el magnetismo animal y la hipnosis como métodos para esclarecer el misterio de un crimen (en muchas ocasiones paranormal), el primer ejemplo de este tipo de detectives aparece en The Experiences of Flaxman Low (1899) de HeskethPrichard, aunque es a comienzos del siglo XX cuando más detectives magnetizadores e hipnotizadores vamos a encontrar en la literatura de ficción.

En este caso, he escogido las novelas El escarabajo (1897) de Richard Marsh (1857-1915) y Drácula (1897) de Bram Stoker (1847-1912) porque en las dos aparecen monstruos con poderes hipnóticos y me interesaba especialmente el papel que juegan estos conocimientos en la construcción de la monstruosidad de los personajes. Además, las dos novelas tienen tantos puntos en común (mismo año de publicación, mismo período literario, mismo subgénero literario, mismo argumento principal, etc.) que casi, inevitablemente, propician que vayan juntas en un trabajo de estas características. En este sentido, rastrearé el tratamiento del magnetismo animal y de la hipnosis que aparece en los textos, analizaré si se puede hablar de un uso argumental o ambiental y si los magnetizadores e hipnotizadores que aparecen en las historias se adecúan a los estereotipos arriba mencionados o si por el contrario estamos ante unas narrativas donde el papel del magnetismo animal y la hipnosis es otro.

\section{DE HIPNOSIS Y SOLO HIPNOSIS, DESDE LA PRÁCTICA MÉDICA AL PODER VAMPÍRICO}

Dentro de la amplia variedad de literatura académica que existe sobre Drácula (1897) es habitual encontrar trabajos donde se analiza la novela de Stoker como interpretación de los diversos miedos y preocupaciones de la sociedad victoriana de finales del siglo XIX, lo que Nicholas Daly ha acuñado como "the anxiety theory" (Daly, 1997, p. 184). En este sentido, Carol Senf ha explorado las preocupaciones victorianas en torno a los roles de género y al surgimiento de idearios feministas como los de la New Woman, expresión esta última muy usada por Stoker en su novela (Senf, 1982, p. 33). Por su parte, Christopher Craft ha rastreado en Drácula los discursos sociales imperantes en la sociedad victoriana sobre el sexo y la homosexualidad (Craft, 1990, p. 216); Stephen Arata ha hecho lo mismo con el concepto de raza y ha señalado el pánico de la sociedad inglesa a lo que ha llamado "reverse colonization" (Arata, 1996, p. 621); David Glover ha señalado la desconfianza de la sociedad victoriana ante el extranjero, el inmigrante (Glover, 2018, p. 86); Christine Ferguson ha analizado la aprensión victoriana en torno al uso de la lengua inglesa de forma no uniforme (Ferguson, 2006, p. 131); Dani Cavallaro se ha centrado en los recelos de la clase burguesa inglesa ante la vieja aristocracia rural, en este caso representada por el conde Drácula (Cavallaro, 2002, p. 183); Franco Moretti y Gail Turley han apuntado que el vampiro encarna la ética y los valores capitalistas (Moretti, 1983, p. 91; Turley, 2005, p. 117); en particular, Turley interpreta que la lucha entre Drácula y el grupo de Van Helsing puede entenderse como una batalla por el monopolio de la circulación del capital y el consumo desmedido (Turley, 2005, p. 117); y Victoria Dawson ha visto en la 
novela el miedo a la degeneración, haciendo referencia al concepto popularizado por el libro Degeneración (1982) de Max Nordau (Dawson, 2016, p. 110). La teoría de la degeneración propuesta inicialmente por Bénédict Morel llegó a ser muy influyente en las sociedades europeas de finales del siglo XIX y sus tesis aparecieron recurrentemente en la literatura de ficción de la época (Pick, 1989, p. 172). Stoker no escapa a esta influencia y cita en su novela a Max Nordau y al célebre médico y criminólogo italiano Cesare Lombroso. El conde Drácula necesitaba parasitar y transformar a otros para poder sobrevivir, por lo que la novela refleja el horror a la degeneración entendida esta como contagio sanguíneo y moral, pero al mismo tiempo también muestra los mecanismos de contención y confinamiento de dicha degeneración; es por esto que la obra de Stoker puede verse como un ejemplo perfecto del discurso degenerativo de finales del siglo XIX (Pick, 1989, p. 173). En este sentido, en relación con el género, la sexualidad, la raza y la clase, el conde Drácula es el desviado, el invertido, el otro, el criminal, el anormal, el degenerado, y finalmente, es el compendio de todos estos agregados lo que lo convierte en un monstruo (Halberstam, 1993, p. 334). No obstante, aún es posible añadir un agregado más a la monstruosidad del conde Drácula: sus poderes hipnóticos. Pues digámoslo ya, el conde Drácula no es un villano hipnotizador, aunque como algunos han señalado comparta muchos rasgos con Svengali, el magnetizador de Trilby (Auerbach, 1982, p. 16), sino un monstruo que entre los muchos poderes diabólicos que domina (controla los elementos climáticos, puede transformase en animales, tiene una fuerza sobrehumana, etc.) posee la capacidad para dominar, controlar y subyugar a sus víctimas mediante la hipnosis.

Ya en los primeros capítulos de la novela, Jonathan Harker, el abogado inglés que ha viajado hasta el castillo de Drácula en Transilvania para gestionarle la compra de una propiedad en Londres, se da cuenta de que “...mientras el conde Drácula hablaba, había un no sé qué en su mirada y en su actitud que me hizo recordar que me encontraba prisionero..." (Stoker, 2005, pp. 142-143), y un poco más adelante comprende que "iMe estaban hipnotizando!" (Stoker, 2005, p. 161). Aunque la trama principal de la novela no es la hipnosis y Drácula es mucho más (en su monstruosidad) que el estereotipo del hipnotizador villano (y no es reducible a esta figura), la hipnosis juega un papel central en la historia, pudiendo hablar de un uso argumental de la hipnosis y no de un uso meramente ambiental, accesorio u ornamental. La posición de Stoker ante el magnetismo animal es ambigua: en Drácula siempre habla de hipnosis y nunca de magnetismo animal o mesmerismo (la palabra hipnosis aparece hasta treinta y cuatro veces en el texto, pero no encontramos una sola mención al magnetismo animal o al mesmerismo). Robert Jones cree que lo anterior es toda una declaración de intenciones de Stoker a favor de la hipnosis y de rechazo del magnetismo animal (Jones, 2009, p. 177), mientras que Leah Davydov interpreta que Stoker habla todo el rato de magnetismo animal, aunque se refiera a este como hipnosis (Davydov, 2017, p. 35). Por otra parte, en la última novela de Stoker, titulada La madriguera del gusano blanco $(1911)^{2}$, sí que aparecen ambos términos (mesmerismo e hipnosis): “...por algún tipo de batalla mesmérica o hipnótica” (Stoker, 2011, p. 58), “...alguna extraordinaria facultad mesmérica o hipnótica” (Stoker, 2011, p. 55), “...un poder impregnado con alguna cualidad misteriosa, parcialmente mesmérica, parcialmente hipnótica..." (Stoker, 2011, p. 13). También creo que Stoker nunca rechazó el magnetismo animal, aunque tuvo sus dudas acerca de su creador, Mesmer. Pero, ¿cuáles eran estas dudas? La respuesta la encontraremos en Famosos impostores (1910), un libro de no ficción de Stoker publicado tan solo dos años antes de su muerte en el que realiza unas semblanzas de personajes históricos designados por él como impostores. La parte del libro, titulada Profesionales de la magia, la componen tres capítulos dedicados a Paracelso, Cagliostro y Mesmer. La elección de los dos personajes que acompañan a Mesmer no puede ser casual, pues ambos guardan relación estrecha con el magnetismo animal: Paracelso (14931541) fue uno de los precursores de las ideas de Mesmer (Ellenberger, 1976, p. 90) y Cagliostro (17431795), a quien se ha llamado alquimista, mago, conspirador, masón, espía, estafador y charlatán, fue principalmente un magnetizador, y así se definió a sí mismo en un proceso judicial en su contra asegurando que el magnetismo animal era lo único que explicaba todos sus poderes (Butler, 1997, p. 320). En Famosos impostores, Stoker nos explica sus motivos para aceptar las aplicaciones terapéuticas del magnetismo animal pero, al mismo tiempo, dudar de su creador: "Aunque Franz Anton Mesmer hizo un descubrimiento sorprendente que, después de haber sido probado y empleado en terapéutica durante un siglo, es aceptado como una contribución a la ciencia, él es incluido en la lista de impostores porque, por sólida que sea su teoría, la usó con las formas o rodeado de la at- 
mósfera de la impostura" (Stoker, 2009, p. 86). En general, el tratamiento de Mesmer en Famosos impostores es bastante impreciso, Stoker parece no decidirse a condenarlo totalmente como un charlatán y en ocasiones lo admite como un científico de una época pasada (más oscura) por lo que sus prácticas estrafalarias e ideas erróneas (la teoría del fluido magnético) quedarían así disculpadas (Stoker, 2009, p. 89).

El grupo de protagonistas que se enfrenta al conde Drácula están dirigidos por el doctor Van Helsing que, gracias a sus muchos títulos de doctor, se comporta como un experto detective resolviendo un enigma que solo él puede comprender; en este aspecto, podemos considerar a Van Helsing como un verdadero precursor de los detectives hipnotizadores mencionados en la introducción. Van Helsing y su grupo luchan contra el vampiro usando algunas técnicas extraídas de las supersticiones populares más arcaicas, como son las flores de ajo, los crucifijos de plata y las estacas de madera clavadas en el corazón, junto con todo un elenco de nuevas tecnologías (entiéndase nuevas para finales del siglo XIX) como la máquina de escribir, el fonógrafo, el telégrafo, la cámara fotográfica, los rifles Winchester, los trenes y el teléfono, además de algunos nuevos conocimientos científicos como la hipnosis, la antropología criminal de Lombroso y las transfusiones de sangre. Pero si ellos juntan lo antiguo con lo moderno, lo viejo con nuevo, la ciencia con la superstición, Drácula también lo hace, pues también él usa la hipnosis y al igual que ocurre en la novela La piedra Lunar (1868) de Wilkie Collins, en la que la hipnosis sirve para cometer el crimen y para resolverlo, Drácula hipnotiza a sus víctimas para chuparles la sangre y Van Helsing, el héroe, usa la hipnosis con una de ellas (Mina Harker) para averiguar el paradero del monstruo y poder destruirlo: "Si es capaz, durante el trance hipnótico, de decirnos lo que el conde ve y oye,..." (Stoker, 2005, p. 549). Como vemos, la novela de Stoker no puede ser reducida a los términos propuestos por algunos autores que la han interpretado como una parábola sobre la ciencia y lo moderno venciendo sobre lo oscuro y arcaico pues ambos bandos enfrentados han cruzado esas fronteras: el grupo de Van Helsing mezclando superstición con ciencia y Drácula haciendo uso de la hipnosis científica (Byron, 2007, p. 55). Es por esto que Julio Pérez prefiere hablar de un enfrentamiento entre dos modelos de modernidad (Pérez, 2014, p. 56).

Sin embargo, entre la hipnosis de Van Helsing y la de Drácula encontramos algunas diferencias; Van Hel- sing tiene como referencia en hipnosis al neurólogo clínico y profesor de anatomía patológica francés JeanMartin Charcot (1825-1893) (Stoker, 2005, p. 363) e hipnotiza usando pases de manos y relajando a la persona que va a ser hipnotizada, mientras que Drácula ha adquirido sus poderes hipnóticos en una especie de secta ocultista llamada Escoliomancia (Stoker, 2005 , p. 521) y puede hipnotizar, en un primer momento, incluso solo con la fuerza de su mirada o con su mera presencia para más tarde hacerse obedecer desde la distancia. Roger Luckhurst establece que las tres grandes etapas históricas del magnetismo animal y de la hipnosis están respectivamente influenciadas por Mesmer a partir de 1780; por el médico inglés director del North London Hospital John Elliotson (1791-1868) a partir de 1839; y por Charcot a partir de 1882 y que estas etapas se corresponden a su vez con etapas claramente diferenciadas en la literatura de ficción mesmérica e hipnótica, en la que se reflejan, en mayor o menor grado, los debates científicos en torno a estas materias (Luckhurst, 2000, p. 148). De acuerdo con esto y teniendo en cuenta lo que ha señalado Anne Harrington: "...the work of the Charcot school on hypnosis and hysteria was conceptually linked to a reawakening of interest in the fundamental ideas of biomagnetism as taught by the old mesmerists" (Harrington, 1988, p. 127), algo de la tesis de Luckhurst encontramos en Drácula cuando Van Helsing interpela al doctor Seward a separar el grano de la paja: "Supongo que usted no creerá en la transferencia corporal, ¿ंverdad? Ni en la materialización, ¿no es cierto? $\mathrm{Ni}$ en los cuerpos astrales, ¿iverdad? Ni en la lectura de pensamiento. Ni en el hipnotismo... En eso último sí, dije, Charcot lo ha demostrado con bastante fundamento" (Stoker, 2005, p. 363).

En un momento de la novela Stoker casi llega a hablar de magnetismo animal cuando Van Helsing explica la facilidad innata de Drácula para la hipnosis: "Sin duda hay algo magnético o eléctrico en algunas de estas combinaciones de fuerzas ocultas que favorecen de un modo extraño la vida física; y él llevaba en su interior, desde el principio, algunas de esas grandes cualidades" (Stoker, 2005, p. 545). Sin embargo, insisto en que Stoker llama hipnosis tanto a las técnicas de Van Helsing como a los poderes de Drácula.

Bram Stoker tardó siete años en escribir Drácula. Todas las notas que tomó durante esos años para escribir la novela han sido publicadas bajo el título Bram Stoker's Notes for Dracula A Facsimile Edition (2008). Entre las anotaciones de ideas argumentales, 
esbozos de personajes, fechas y lugares, encontramos bastantes referencias de libros de diversas materias que usó para documentarse, como On the Truths Contained in Popular Superstitions with an Account of Mesmerism (1851) de Herbert Mayo, profesor de anatomía y fisiología en King's College de Londres, que dedica capítulos al sonambulismo, a los estados de trance, al vampirismo y al mesmerismo, temas todos ellos usados en la creación de Drácula (Stoker, 2008, p. 305). Otra de las influencias mesméricas para Stoker fue su gran amigo Hall Caine (Hopkins, 2007, p. 24), a quien va dedicada la novela bajo el apodo de Hommy Beg. Algunos autores han afirmado que Caine tuvo un papel importante en la escritura de Drácula, ya sea como negro (ghost writer) o como corrector del texto (McNally, 1975, p. 135). Lo cierto es que Caine había publicado una novela por entregas titulada Drink: A Love Story on a Great Question (1890) en la que un hipnotizador francés llamado La Mothe es contratado por el novio de una mujer llamada Lucy (la primera víctima de Drácula también se llama Lucy) para que la cure de su alcoholismo (Skal, 2017, p. 314). En la obra de Caine destacan algunos hechos muy interesantes: es una de las pocas novelas donde el hipnotizador no es un villano sino un hombre decente que consigue curar a su paciente mediante la hipnosis; además, separa claramente los conceptos del magnetismo animal y la hipnosis como prácticas diferentes: "La Mothe seemed to guess at the nature of my objection, for he began to argue the claims of hypnotism as distinguished from those of mesmerism" (Caine, 1907, p. 40).

Es una incógnita por qué Stoker no usó la palabra mesmerismo o magnetismo animal en Drácula. Tal vez fuera por sugerencia de Hall Caine, que entendió que lo adecuado era usar solo esa palabra. También es posible que Stoker se dejara influir por el destino de Herbert Mayo, su fuente en torno a estas materias, que tuvo que abandonar su cátedra y altos cargos exiliándose a Alemania por el descrédito que le proporcionaron sus trabajos sobre el mesmerismo (López-Piñero; Morales, 1970, p. 124).

\section{EL ESCARABAJO DE MARSH, UN MONSTRUO INDEFINIDO CON UN PODER AMBIGUO}

La novela de Richard Marsh El escarabajo fue comparada desde el mismo momento de su aparición con Drácula. Los motivos de esta comparación hay que buscarlos en que ambas novelas fueron publicadas el mismo año y que las dos gozaron de un gran éxito de ventas y de numerosas reediciones (Drácula hasta la actualidad y El escarabajo hasta mediados del siglo $X X)$, además ambas comparten algunos rasgos argumentales: los protagonistas son ingleses de clase alta que deben enfrentarse a un monstruo extranjero con poderes hipnóticos que ha llegado a Londres con propósitos siniestros (Jones, 2011, p. 66). Otra similitud entre Drácula y El escarabajo es que están escritas siguiendo la misma técnica: la novela de Marsh está estructurada en cuatro partes, cada una de ellas narrada por un personaje diferente que va aportando su particular punto de vista, mientras que Drácula está contada alternando los diarios y anotaciones de los diferentes protagonistas ${ }^{3}$. Parece ser que esta técnica narrativa se puso de moda en el período del gótico tardío victoriano; otros escritores que la emplearon fueron Wilkie Collins en la ya citada La piedra lunar (1868) y Arthur Machen en Los tres impostores (1895).

Las primeras comparaciones entre Drácula y El escarabajo vinieron de mano de algunos críticos literarios de finales del siglo XIX. En estas reseñas comprobamos que las novelas eran tratadas por igual; algunos críticos se decantaban por la novela de Stoker y otros por la de Marsh (Vuohelainen, 2006, p. 94). Sin embargo, en la actualidad Drácula es un clásico literario y el personaje del conde un fenómeno de masas, mientras que El escarabajo es una novela olvidada y desconocida. En este sentido, vamos a encontrar referencias a Drácula en cualquier trabajo académico sobre El escarabajo, pero no al revés; el equilibrio entre las novelas se ha roto y difícilmente encontraremos una mención a El escarabajo en un trabajo sobre Drácula.

Siguiendo la "anxiety theory" antes mencionada es lógico que tanto Drácula como El escarabajo puedan analizarse como reflejos, simbólicos o literales, de los temores de la sociedad victoriana de finales del siglo XIX. En este sentido, El escarabajo es un muestrario, en mayor o menor grado, de los mismos miedos, obsesiones y preocupaciones que aparecen en Drácula (raza, clase, género, sexualidad, "reverse colonization", etc.). Sin embargo, en El escarabajo encontramos algunos recelos particulares que no localizamos en el texto de Stoker: la incomodidad de la clases altas ante el crecimiento desmesurado de la ciudad de Londres con los peligros inherentes a las grandes metrópolis (Vuohelainen, 2006, p. 97), la intolerancia de los ricos ante la pobreza y el miedo a la crimina- 
lidad ligada a esta (Generari, 2012, p. 37), la repulsión a las plagas de insectos foráneos y a sus posibles consecuencias dañinas (Effinger, 2017, p. 257) y un temor paranoico a las enfermedades de transmisión sexual provenientes de las colonias y propagadas por los soldados ingleses (Hurley, 1996, p. 127).

En El escarabajo, el monstruo no está claramente definido, Julian Wolfreys lo ha Ilamado "beetle-human hybrid" (Wolfreys, 2007, p. 31) y Roger Luckhurst "liminal man-woman-goddess-beetle-Thing" (Luckhurst, 2000, p. 160), lo que queda claro es que se trata de un ser de una fealdad monstruosa: aunque es mujer se disfraza como un hombre y adquiere aspecto y rasgos de árabe; puede transformase en escarabajo y cuando se nos presenta con envoltura humana habla de una forma extraña produciendo los zumbidos típicos de los insectos; además posee una fuerza sobrehumana y domina las artes mesméricas o hipnóticas. En El escarabajo, Marsh usa los dos términos, mesmerismo e hipnotismo, como sinónimos, sin diferencias de ningún tipo; en un momento puede usar la expresión "the mesmeric quality" y solo unas líneas después usar "hypnotic" para hablar de lo mis-

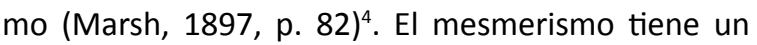
papel argumental en El escarabajo y está muy presente durante toda la novela. Es casi el único poder paranormal del monstruo (Drácula poseía muchos más), pero al igual que Drácula el monstruo de El escarabajo no puede ser reducido al estereotipo del magnetizador villano, pues el magnetismo animal es un elemento más para cimentar su monstruosidad. Marsh explotaría el recurso del mesmerismo y de la hipnosis en bastantes de sus creaciones literarias ${ }^{5}$; el tratamiento de los términos "mesmerismo" e "hipnosis" como sinónimos es el mismo en todas sus ficciones mesméricas. Su postura queda sintetizada en el relato By Suggestion (1900): “Hypnotism! Isn't that the new-fangled word for mesmerism? Precisely" (Marsh, 1900, p. 185).

El monstruo de Marsh, al que llamaré "el escarabajo" de aquí en adelante, ha llegado a Londres para vengarse de Paul Lessingham, un popular político reformista, por algún acto que este cometió en $\mathrm{El}$ Cairo durante su juventud. El escarabajo mesmeriza a un vagabundo llamado Robert Holt para que le ayude en sus propósitos. Holt, narrador de la primera parte del libro, nos dice que: "Nunca antes me había dado cuenta de lo que significaba el poder de la mirada. Sus ojos me mantenían encadenado, desvalido, hechizado. Sentí que podían hacer conmigo lo que quisieran, y así lo hicieron" (Marsh, 2018, p. 51); y unas páginas después: "Hizo un movimiento con la mano y, de inmediato, pasó lo mismo que la noche anterior; tuvo lugar una metamorfosis en los mismísimos abismos de mi ser" (Marsh, 2018, p. 62); después de la mirada fija y poderosa y los pases de manos, Holt termina concluyendo lo obvio: “...me di cuenta de que ejercía sobre mí un grado de fuerza hipnótica [mesmeric] que jamás imaginé que una criatura pudiera ejercer sobre otra" (Marsh, 2018, p. 62). A partir de aquí Holt pasa a estar bajo el poder del escarabajo, que lo va a manejar como a una marioneta o como a un autómata manejado por control remoto.

Sin embargo, los poderes magnéticos del escarabajo no son omnipotentes, ya que intenta hipnotizar sin éxito a Sydney Atherton, un inventor de armas químicas de destrucción masiva. Atherton, que es el narrador de la segunda parte del libro, nos dice que: "Carezco por completo de esa sensibilidad que posee el sujeto hipnotizado" (Marsh, 2018, p. 121); y cuando el escarabajo trata de hipnotizarlo: "Sin embargo, mientras seguía contemplando a ese individuo, era consciente de que solo gracias a la fuerza de voluntad podía resistir aquel hilo siniestro que parecía estar pasando desde sus ojos a los míos" (Marsh, 2018, p. 170). Atherton consigue asustar al escarabajo con descargas eléctricas y con vapores producidos por bromuro de fósforo en un pasaje que podría fácilmente interpretarse como trasunto literario de la comisión real designada por Luis XVI de Francia, la cual dictaminó en contra del magnetismo animal en 1784 y de la que formaban parte entre otros Benjamin Franklin (1706-1790), famoso por sus experimentos con la electricidad, y Antoine Laurent de Lavoisier (1743-1794), reconocido químico (Engelhardt, 2003, p. 65). Finalmente, como ya he apuntado, el escarabajo no consigue hipnotizar a Atherton, y es del propio texto de donde pueden extraerse hasta tres explicaciones, no excluyentes, para la resistencia de este a la hipnosis. La primera ya ha sido expuesta antes: Atherton, por su temperamento, es un sujeto difícilmente hipnotizable y, además, las demostraciones de electricidad y química han roto el influjo hipnótico del escarabajo. Una segunda explicación es que Atherton también posee el poder de la hipnosis y por tanto es inmune a ella, como Marjorie Lindon, la narradora de la tercera parte del libro, nos dice de Atherton: "He oído decir que posee un poder hipnótico hasta grados inusitados y que, si decidiera ejercerlo, podría llegar a ser un peligro para la sociedad. 
Creo que ha hipnotizado a Dora" (Marsh, 2018, p. 237). La tercera explicación, que tal vez sea la más interesante de todas, es que los poderes hipnóticos del escarabajo solo funcionan con personas que se encuentran en un estado de debilidad física y mental o con personas histéricas. De este modo, el escarabajo puede hipnotizar a Holt, que lleva varios días sin comer nada y se encuentra en un estado de agotamiento próximo a la muerte; el mismo Holt lo explica: "Supongo que la tensión y las privaciones que había padecido últimamente y que, inlcuso en esos momentos, seguía padeciendo, tuvieron mucho que ver con mi reacción de entonces y con mi comportamiento en todo lo que siguió" (Marsh, 2018, p. 45). De forma parecida, Paul Lessingham le cuenta al detective Auguste Champnell que: "Solo puedo suponer que, durante aquellas semanas, ella me mantuvo allí en un estado de estupor hipnótico [mesmeric]. Que, aprovechándose de la debilidad que la fiebre había dejado en mí, mediante las prácticas de sus artes diabólicas, me había impedido salir de aquel trance hipnótico" (Marsh, 2018, p. 308).

Como apuntaba antes, las alusiones a las personalidades histéricas de Holt y Lessingham son frecuentes en el texto, Marjorie nos dice sobre Holt: “...las aventuras misteriosas de aquel pobre, histérico y débil metal que era el señor Holt..." (Marsh, 2018, p. 287); y Champnell sobre Lessigham: "...este líder de hombres, cuya principal característica en la Cámara de los Comunes era la entereza, estaba transformándose en una mujer histérica" (Marsh, 2018, p. 377). Esta relación entre la histeria y la hipnosis conecta la narración con una de las conclusiones de Charcot sobre estas materias; en concreto, que el trance hipnótico debe ser entendido como una "neurosis provocada" y que solo las personas histéricas pueden ser hipnotizadas (López-Piñero, 2002, p. 56). Sin embargo, el detective Champnell también hace referencia a la hipnosis por sugestión al hablar de Holt: "También parece hipnotizado. Si es así, debe ser por sugestión... y eso es lo que me hace dudar, porque sería el primer caso claramente probado de hipnotismo por sugestión con el que me he topado..." (Marsh, 2018, p. 281). Parece ser que Marsh está haciendo referencia a las teorías del profesor de la Clínica Médica de Nancy Hippolyte Bernheim (1840-1919) sobre la hipnosis por sugestión, y en particular a la posibilidad de que un sujeto que ha sido previamente hipnotizado pueda responder a sugerencias similares a las dadas en estado hipnótico una vez despierto (Bernheim, 1886, p. 77). De esta forma, Marsh, tal vez sin ser consciente de ello, trasladaba al personaje del hipnotizado Holt una de las controversias más famosas dentro de la historia del hipnotismo: el enfrentamiento de Charcot y la escuela de la Salpêtrière (como defensores de la "neurosis provocada") con Bernheim y la escuela de Nancy (como defensores del hipnotismo por sugestión). Para la escuela de la Salpêtrière la sugestión era una manifestación más de la hipnosis; en cambio, para la escuela de Nancy era el mecanismo básico que la explicaba (López-Piñero; Morales, 1970, p. 194). En el relato By Suggestion, anteriormente citado, Marsh nos da otro ejemplo de lo que entiende por hipnosis por sugestión: "I shall put this note into an envelope; I shall send it round by my boy, and when she opens it and reads it she will be hypnotized. We call it hypnotism by suggestion" (Marsh, 1900, p. 152), algo totalmente posible según Bernheim: "Se las puede hipnotizar por carta, afirmándoles, por ejemplo, que inmediatamente que concluyan su lectura se dormirán" (Bernheim, 1886, p. 5).

Otro pasaje interesante sobre los usos de la hipnosis en la novela de Marsh es cuando mediante la hipnosis el escarabajo cura a un amigo de Atherton que está agonizante. Atherton le pregunta: “¿Lo ha hipnotizado?", y el escarabajo le responde: "¿Y qué más da?" (Marsh, 2018, p. 166). El mensaje de Marsh me parece nítido y bastante revelador de su postura: ¿Qué importancia tiene cómo se llame algo que puede salvar a un hombre de la muerte? ¿Dejará de ser menos real si lo llamamos magnetismo animal o mesmerismo? ¿Cambiará el hecho de la curación si lo llamamos hipnotismo? ¿No son más importantes los hechos atribuidos a un fenómeno que los nombres teóricos con los que se intenta designar a ese fenómeno?

Tan solo un año después de la publicación de El escarabajo Marsh publicaría otra ficción mesmérica, la novela The house of Mystery (1898), en la que aparece un malvado hipnotizador llamado Aaron Lazarus que es inglés, burgués y de raza blanca. En esta ocasión, Marsh despojó de monstruosidad a su villano, tan solo mantuvo en él los poderes hipnóticos. En este sentido, Lazarus ya no podía reflejar en su totalidad las ansiedades de la sociedad victoriana de fin de siglo: tenía que ser un monstruo para poder hacerlo y Lazarus era solo un villano hipnotizador. 


\section{CONCLUSIONES}

Tanto Stoker como Marsh hicieron un uso argumental del mesmerismo y de la hipnosis en sus más famosas novelas, y ambos encarnaron en sus monstruos, pretendiéndolo o no, los principales miedos y preocupaciones de la sociedad victoriana de finales del siglo XIX, como los miedos al extranjero, al homosexual, al pobre, a las enfermedades venéreas, a los idearios feministas, a la degeneración racial y moral del individuo, a la colonización inversa, etc. Los dos autores dotaron a sus terribles criaturas del poder de la hipnosis, pero ni el escarabajo de Marsh ni el vampiro de Stoker pueden ser reducidos por ello al clásico estereotipo de magnetizador/hipnotizador villano, ya que en su monstruosidad lo sobrepasan, aunque comparten con este lo que podría llamarse una "goticización del magnetismo animal". En realidad, la hipnosis y el magnetismo animal funcionan aquí como agregados que, junto con la desviación sexual, la otredad, la criminalidad, la anormalidad, la fealdad y la degeneración racial terminan por conformar la monstruosidad de los personajes.

Por otra parte, casi podría parecer natural que unos seres como el escarabajo y Drácula dominen los poderes del mesmerismo y de la hipnosis; unos poderes que, como los monstruos que los detentan, escapan a una definición sencilla ya que se mueven en territorios fronterizos: son poderes humanos y cósmicos, científicos y mágicos, terapéuticos y dañinos, liberadores y esclavizadores.

La postura de Stoker y Marsh ante el mesmerismo y la hipnosis también coincide en su ambigüedad: en las dos novelas la hipnosis es usada para hacer el bien y el mal. En Drácula tanto Van Helsing como el vampiro usan la hipnosis y en El escarabajo es el mismo monstruo (algo que añade complejidad al personaje) el que salva la vida a un amigo de Atherton mediante la hipnosis. Esta ambivalencia en torno al uso de la hipnosis, que era practicada tanto por prestigiosos médicos como por charlatanes de dudosa reputación y era percibida como un poder peligroso, pero al mismo tiempo como una fuerza curativa, fue un rasgo típico de la sociedad inglesa de finales del siglo XIX (Winter, 1998, pp. 6-8).

En Drácula, Stoker usa solo la palabra "hipnosis" tanto para referirse a los poderes del héroe como a los del monstruo, albergaba sus reticencias hacia Mesmer, pero no hacia el magnetismo animal como práctica terapéutica. En cambio, en La madriguera del gusano blanco maneja los dos términos (mesmerismo e hipnosis), aunque de una forma ambigua. Por su parte, Marsh usó los términos "mesmerismo" e "hipnosis" como sinónimos en todas sus ficciones mesméricas pero introdujo matices muy interesantes en torno a estas materias, como que solo las personas que se encuentran en un estado de debilidad física y mental o que tienen personalidades histéricas pueden ser hipnotizadas, siguiendo las teorías de Charcot sobre la hipnosis como proceso patológico; o como la hipnosis por sugestión, siguiendo las teorías de Bernheim.

Drácula y El escarabajo no pueden ser reducidas, como pretenden algunos autores, a una lucha entre lo arcaico y lo moderno, entre la magia y la ciencia, entre lo racional y lo irracional, ya que todas estas fronteras son franqueadas innumerables veces por todos los personajes que aparecen en ambas novelas, tanto por los héroes como por los monstruos. Es así como Van Helsing, el científico, el varias veces doctor, cree en las más antiguas supersticiones en torno a los vampiros (Stoker, 2005, p. 365); por su parte, Atherton, el inventor, el químico, se llama a sí mismo mago y a su laboratorio cueva de hechicero (Marsh, 2018 , p. 175). Los monstruos tampoco respetan los límites establecidos por el dogma cientificista de la época y se apropian de la ciencia de la hipnosis, pues a pesar de que sus poderes son mucho más antiguos que el descubrimiento del magnetismo animal por parte de Mesmer, en los dos textos se presentan vestidos con los ropajes de la hipnosis científica.

Finalmente, los monstruos son perseguidos y derrotados, Drácula muere atravesado por una estaca de madera a las puertas de su Castillo, donde pretendía esconderse, y el escarabajo muere en un fortuito accidente de tren mientras intentaba huir. Si el monstruo es un constructo y una proyección de los miedos sociales (Cavallaro, 2002, p. 171), su muerte se convierte en un exorcismo; no hay un intento de comprensión por parte de los humanos de tales criaturas, ni siquiera un intento de estudiarlos científicamente, solo un ansia desmedida de aniquilación. Es la misma postura que sufrió el magnetismo animal primero y después la hipnosis, en primer lugar, fascinación y temor, después persecución y aniquilación. Tal vez podríamos reprochar a Drácula y a El escarabajo que, como novelas, como artefactos textuales, como fuentes de conocimiento, también han contribuido, en parte, en esa persecución a muerte del mesmerismo y de la hipnosis. No es inocente pensar que la asociación de estos po- 
deres con la figura del monstruo, al igual que con el estereotipo del villano gótico, podría tener un efecto de deslegitimación de estas prácticas tanto en la sociedad como en la comunidad médica de su tiempo (González De Pablo, 2003, p. 236), aunque ambos autores dejen la puerta abierta en sus textos,

\section{NOTAS}

1 Aparece en dos relatos de Emilio Carrere titulados Embrujamiento (1918) y Lo que vio la reina de Francia (1919).

2 Se recomienda la lectura de la primera edición de la novela en 1911, en la segunda edición el texto fue considerablemente reducido pasando de unas 300 páginas a menos de la mitad y así es como ha continuado reeditándose hasta la actualidad.

3 La famosa novela de Mary Shelley Frankenstein o el moderno Prometeo (1818) ya anticipaba este estilo al estar narrada a través de cartas, aunque aquí siempre es la misma voz narrativa.

\section{BIBLIOGRAFÍA}

Auerbach, Nina (1982), Woman and the Demon, the Life of a Victorian Myth, Cambridge, Harvard University Press.

Arata, Stephen (1996), "The Occidental Tourist: Dracula and the Anxiety of Reverse Colonization", Victorian Studies, 33, (4), pp. 621-645.

Bernheim, Hippolyte (1886), De la sugestión y de sus aplicaciones en terapéutica, Oviedo, Imprenta de Vicente Brid.

Butler, Eliza (1997), El mito del mago, Cambridge, Cambridge University Press.

Byron, Glennis (2007), "Bram Stoker's Gothic and the Resource of Science", Critical Survey, 19, (2), pp. 48-62.

Caine, Hall (1907) [1890], Drink: A Love Story on a Great Question, New York, Appleton and Company.

Cavallaro, Dani (2002), The Gothic Vision: Three Centuries of Horror, Terror and Fear, London, Bloomsbury Publishing.

Craft, Christopher (1990), "Kiss Me with Those Red Lips: Gender and Inversion in Dracula". In: Showalter, Elaine (ed.), Speaking of Gender, New York, Routledge, pp. 216-242.

Daly, Nicholas (1997), "Incorporated Bodies: Dracula and the Rise of Professionalism", Texas Studies and Literature and Language, 39, (2), pp. 181-203.

Darnton, Robert (1968), Mesmerism and the end of the Enlightenment in France, Cambridge, Harvard University Press. como ya se ha dicho, a las bondades curativas y benéficas del mesmerismo y la hipnosis. Si nos decidiéramos a reprocharles lo anterior, tendríamos que reconocer que, paradójicamente, Drácula y EI escarabajo también han salvado del olvido y de la aniquilación total a aquello que perseguían.

4 Uso aquí la versión original en inglés para referirme al uso de los términos "mesmerismo" e "hipnosis" ya que en la traducción al español aparecen sistemáticamente ambos términos traducidos como "hipnosis". En las citas siguientes de la novela pongo entre corchetes el término que aparece en el original en inglés al lado de la traducción al español.

5 Lo haría en las novelas: The House of Mystery (1898) y The Woman with One Hand (1899); y en los relatos: An Experience (1900), By Suggestion (1900), How He Passed (1900), A Double-Minded Gentleman (1900), The Strange Occurrences in Canterstone Jail (1901) y His First Experiment (1901).

Davydov, Leah (2017), “Only a Sufficient Cause: Bram Stoker's Dracula as a Tale of Mad Science and Faustian Redemption", ETD Archive, 953, pp. 1-66.

Dawson, Victoria (2016), “Gay Motes that People the Sunbeams: Dust, Death and Degeneration in Dracula". In: Wynne, Catherine (ed.), Bram Stoker and the Gothic Formations to Transformations, New York, Palgrave Macmillan, pp. 105-117.

Effinger, Elizabeth (2017), "Insect Politics in Richard Marsh's The Beetle". In: Mazzeno, Laurence; Morrison, Donald (eds.), Animals in Victorian Literature and Culture, London, Palgrave Macmillan, pp. 251-268.

Ellenberger, Henri (1976), El descubrimiento del inconsciente, Madrid, Editorial Gredos.

Engelhardt, Dietrich (2003), “Mesmer en la ciencia natural y en la medicina del romanticismo". En: Montiel, Luis; González de Pablo, Ángel (coord.), En ningún lugar, en parte alguna. Estudios sobre la historia del magnetismo animal y del hipnotismo, Madrid, Frenia, pp. 63-100.

Ferguson, Christine (2006), Language, Science and Popular Fiction in the Victorian Fin-de-Siècle, Aldershot, Ashgate.

Gauld, Alan (1992), A History of Hypnotism, New York, Cambridge University Press.

Generari, Gustavo (2012), "The Beetle: A Rhetoric Betrayed". In: Marks, Peter (ed.), Literature and Politics: Pushing the World in Certain Directions, Newcastle, Cambridge Scholars Publishing, pp. 35-45. 
Glover, David (2018), "Dracula in the Age of Mass Migration". In: Luckhurst, Roger (ed.), The Cambridge Companion to Dracula, New York, Cambridge University Press, pp. 85-94.

González De Pablo, Ángel (2003), “El hipnotismo en la España del primer tercio del siglo XX". En: Montiel, Luis; González de Pablo, Ángel (coord.), En ningún lugar, en parte alguna. Estudios sobre la historia del magnetismo animal y del hipnotismo, Madrid, Frenia, pp. 229-300.

Halberstam, Judith (1993), "Technologies of Monstrosity: Bram Stoker's Dracula", Victorian Studies, 35, (3), pp. 333-352.

Harrington, Anne (1988), "Hysteria, Hypnosis, and the Lure of the Invisible: The Rise of Neo-mesmerism in fin-de-siècle French Psychiatry". In: Bynum, William; Porter, Roy; Sheperd, Michael (eds.), The Anatomy of Madness: Essays in the History of Psychiatry vol.3, London, Taylor and Francis, pp. 226-246.

Hartman, Donald (1987), "Hypnotic and Mesmeric Themes and Motifs in Selected English-Language Novels, Shorts Stories, Plays and Poems, 1820-1983", Bulletin of Bibliography, 44, (3), pp. 156-166.

Hopkins, Lisa (2007), Bram Stoker: A Literary Life, Basingstoke, Palgrave Macmillan.

Hurley, Kelly (1996), The Gothic Body Sexuality, Materialism, and Degeneration at the Fin de Siècle, Cambridge, Cambridge University Press.

Jones, Anna (2011), "Conservation of Energy, Individual Agency, and Gothic Terror in Richard Marsh's The Beetle, or, What's Scarier Than an Ancient, Evil, Shape-Shifting Bug?", Victorian Literature and Culture, 39, (1), pp. 65-85.

Jones, Robert (2009), "Mesmerism and Magic, Science and Self in The Beetle and Dracula", Innervate, (1), pp. 175-181.

López-Piñero, José María; Morales, José María (1970), Neurosis y psicoterapia: Un estudio histórico, Madrid, Espasa-Calpe.

López-Piñero, José María (2002), Del hipnotismo a Freud. Orígenes históricos de la psicoterapia, Madrid, Alianza Editorial.

Luckhurst, Roger (2000), "Trance-Gothic 1882-87". In: Robbins, Ruth; Wolfreys, Julian (eds.), Victorian Gothic Literary and Cultural Manifestations in the Nineteenth Century, New York, Palgrave, pp. 148- 167.

Luckhurst, Roger (2002), The Invention of Telepathy: 18701901, Oxford, Oxford University Press.

Macnally, Raymond; Florescu, Radu (1975), In Search of Dracula: The History of Dracula and Vampires, New York, Houghton Mifflin Company.

Marsh, Richard (1897), The Beetle: A Mystery, London, Fisher Unwin.

Marsh, Richard (1900), "By Suggestion". In: Marvels and Mysteries, London, Methuen and Company, pp. 152-193.

Marsh, Richard (2018) [1897], El escarabajo, Madrid, Valdemar.
Mayo, Herbert (1851), On the Truths Contained in Popular Superstitions with an Account of Mesmerism, London, William Blackwood and Sons.

Montiel, Luis (2003), "Primera mirada sobre el lado oscuro del magnetismo: El magnetizador (1813) de E.T.A Hoffmann". En: Montiel, Luis; González de Pablo, Ángel (coords.), En ningún lugar, en parte alguna. Estudios sobre la historia del magnetismo animal y del hipnotismo, Madrid, Frenia, pp. 143-170.

Moretti, Franco (1983), Signs Taken as Wonders: On the Sociology of Literary Forms, London, Verso.

Pérez, Julio (2014), Drácula Superstar, Málaga, Ediciones Corona Borealis.

Pick, Daniel (1989), Faces of Degeneration, Cambridge, Cambridge University Press.

Purcell, Edward (1977), "Trilby and Trilby-Mania: The Beginning of the Bestseller System", Journal of Popular Culture, 11, (1), pp. 62-76.

Quiller-Couch, Arthur (1890), "A literary causerie: Hypnotic fiction", The Speaker: A Review of Politics, Letters, Science and the Arts, 14, p. 316.

Senf, Carol (1982), "Dracula: Stoker's Response to the New Woman", Victorian Studies, 26, (1), pp. 33-49.

Skal, David (2017), Algo en la sangre la biografía secreta de Bram Stoker el hombre que escribió Drácula, Madrid, Es Pop Ediciones.

Stoker, Bram (2005) [1897], Drácula, Madrid, Cátedra.

Stoker, Bram (2008), Bram Stoker's Notes for Dracula a Facsimile Edition, London, McFarland and Company.

Stoker, Bram (2009) [1910], Famosos impostores, Santa Cruz de Tenerife, Melusina.

Stoker, Bram (2011) [1911], La madriguera del gusano blanco, Madrid, Valdemar.

Turley-Houston, Gail (2005) "Bankerization panic and the corporate personality in Dracula". In: Turley-Houston, Gail, From Dickens to Dracula. Gothic, Economics, and Victorian Fiction, Cambridge, Cambridge University Press, pp. 112-131.

Vuohelainen, Minna (2006), “Richard Marsh's The Beetle (1897): a late Victorian popular novel", Working with English: Medieval and Modern Language, Literature and Drama, 2, (1), pp. 89-100.

Winter, Alison (1998), Mesmerized: Powers of Mind in Victorian Britain, Chicago, The University of Chicago Press.

Wolfreys, Julian (2007), "The hieroglyphic other: The Beetle, London and the anxieties of late Imperial England". In: Wolfreys, Julian, Writing London. Volume 3: Inventions of the City, Basingstoke, Palgrave Macmillan, pp. 8-36. 
